\title{
COOPERACIÓN INTERNACIONAL Y FINANCIAMIENTO DE ACTIVIDADES DE DESARROLLO TECNOLÓGICO EN CоLомBia
}

\author{
Fredy Alexander Gómez Jiménez ${ }^{1}$ \\ Jorge Robledo Velásquez ${ }^{2}$
}

\section{Resumen}

Este trabajo presenta los resultados de un estudio de caracterización sobre las relaciones que firmas establecidas en Colombia tienen con instituciones internacionales alrededor del mundo, y al mismo tiempo analiza sus consecuencias en la política pública y en la gestión de las empresas. Particular atención se presta a la Actividades de Desarrollo Tecnológico (ADT), la cual incluye Tecnologías Incorporadas al Capital, Tecnologías de Gestión, Tecnologías Transversales, Aprendizaje Tecnológica e I+D. Adicionalmente, se realiza un estudio comparativo entre aquellas firmas tienen vínculos internacional para la financiación de ADT y aquellas que carecen de ello. Este estudio incluye metodologías de Análisis Multivariantes de datos como Análisis de Factor y Análisis de Clúster. Los datos se obtuvieron de la Segunda Encuesta de Innovación en Colombia, la cual se aplicó en el 2005 a una muestra de 6222 firmas. Entre los diferentes resultados encontrados, se obtuvo una escasa participación que suele tener las

1 Ingeniero Industrial (Universidad Nacional de Colombia Sede Medellín), Máster en Ingeniería Administrativa (C) (Universidad Nacional de Colombia), Docente Ocasional de Tiempo Completo. Instituto Tecnológico Metropolitano. fredygomez@itm.edu.co

2 Ingeniero Mecánico (Universidad Nacional de Colombia Sede Medellín), Magíster en Sistemas de Generación de Energía Eléctrica (Universidad del Valle, Colombia), Ph.D en Estudios de Política y Gestión de Ciencia y Tecnología (Universidad de Sussex, Inglaterra), Profesor Asociado, Facultad de Minas, Universidad Nacional de Colombia Sede Medellín. jrobledo@unal.edu.co 
firmas establecidas en Colombia para el financiamiento de ADT, con un porcentaje que no supera el $1 \%$ del total de la muestra.

\section{Palabras Claves:}

Cooperación Internacional, Actividades de Desarrollo Tecnológico, Análisis Multivariante.

\section{Abstract}

This work presents the outcomes of a characterization study about the relations that firms established in Colombia set up with international institutions from around the world (Europe, United States and Latin America), and at the same time, it analyses their implications for public policy and enterprise management. Particularly attention deserves the funding of Technological Development Activities (TDA), which include Capital Incorporated Technologies, Management Technologies, Transversal Technologies, R\&D and Technological learning. This work also carries out a comparative study between firms that have international links for funding TDA and those that do not have this international links. This study includes data analyses using Multivariate Analysis such as Factor and Cluster Analysis. Data were obtained from the Second Colombian Innovation Survey, which was applied in 2005 to a sample of 6222 firms. Among other results, the study shows that financial resources for firms' R\&D activities come mainly from national sources, with an important participation of international financial resources for Technological Learning Activities. Regard to the firms that have participated in international funding of TDA, the study found that only $1 \%$ of the total firms have employed at least one funding source, showing a clear evidence of the lack of international participation of the studied firms.

\section{Key words:}

International Cooperation, Technology Developments Activities, Multivariate Analysis. 


\section{INTRODUCCIÓN}

La cooperación internacional de tipo empresarial en actividades de Ciencia y Tecnología (CyT) ha sido una actividad que tradicionalmente se ha llevado a cabo principalmente entre organizaciones de países desarrollados, e inclusive hoy en día, esta actividad continúa siendo muy fuerte. Sin embargo, un importante crecimiento de este tipo de relacionamiento ha tomado forma en aquellos países llamados en desarrollo. De acuerdo a la OCDE (2008) y particularizando a las actividades de Investigación y Desarrollo Experimental (I+D), las empresas extranjeras ubicadas en países en desarrollo incrementaron sus gastos de I+D entre la mitad y tres veces la cantidad invertida en el país de origen, y aunque los gastos aún son modestos, su crecimiento es muy relevante. En otro estudio presentado por la Comisión Europea (European Commission, 2007), se encontró que los flujos de gastos de I+D, los cuales solían estar dirigidos hacia países desarrollados, parecen estar virando a favor de las economías emergentes.

Varias de las razones que explican este fenómeno de desplazamiento de capitales están asociadas a la intensificación de la competencia global, los rápidos cambios tecnológicos, el acceso al personal de I+D en países en desarrollo y el aprovechamiento de la diferencia de costos entre los países en desarrollo y desarrollados (Reddy, 1997). Richards \& Yang (2007) establecen que las Corporaciones Multinacionales (CMN), las cuales solían enfocar sus actividades de I+D solo en países desarrollados (Katz et al., 1996, Vonortas, 1997), están ahora comenzando a invertir en países en desarrollo dado que la infraestructura para realizar actividades de investigación ha mejorado, los mercados locales están creciendo y los costos de I+D son considerablemente bajos.

De todas maneras, los efectos generales actuales de la globalización, como la estandarización de los productos de forma global y las facilidades de comunicación alrededor del mundo, han facilitado enormemente la globalización de actividades de I+D y de Capacitación Tecnológica. Adicionalmente, los cambios del mercado 
laboral en países en desarrollo ha incrementado considerablemente el atractivo de establecer filiales en estos países.

En Colombia, la internacionalización de las actividades de CyT ha sido un componente tradicional de la política pública. En la actualidad, la política vigente tiene como objetivo fundamental el de consolidar la proyección internacional del país en esta dimensión, facilitando a los centros de I+D y grupos relacionados el acceso a los recursos financieros e intelectuales internacionales, buscando favorecer la inserción de la ciencia, la tecnología y la innovación colombianas en el contexto internacional (Colombia COLCIENCIAS, 2008). Para poder alcanzar este objetivo, se contemplan, como líneas de acción: la búsqueda y utilización de fuentes internacionales de cooperación; el soporte a la movilidad de investigadores e innovadores; el aprovechamiento de la diáspora en CyT; y el desarrollo de la cooperación horizontal con otros países Caribeños y de Latinoamérica.

El interés del presente estudio se centra en el acceso a recursos financieros internacionales por parte de las empresas establecidas en el país, como una medida del grado de internacionalización de las actividades empresariales de innovación y desarrollo tecnológico, y como un aporte al diagnóstico de la realidad colombiana en esta materia de interés político público. En este sentido, el estudio busca hacer una contribución al conocimiento de un fenómeno que ha sido muy poco estudiado en Latinoamérica (Grosse, in press). En particular, el trabajo estudia las características de la financiación internacional de las Actividades de Desarrollo Tecnológico (ADT) realizadas por las empresas establecidas en el país, según se desprende de los resultados de la Segunda Encuesta de Innovación colombiana.

La estructura del trabajo es como sigue: en la próxima sección se introducen las definiciones y clasificaciones que utiliza la Segunda Encuesta de Innovación para el tratamiento del fenómeno bajo estudio; en la sección 3 se presenta la metodología utilizada, basada en técnicas descriptivas para realizar un primer examen exploratorio de los datos y en Técnicas de Análisis Multivariante (TAM), para la reducción de variables y la identificación y 
caracterización de grupos de empresas similares; en la sección 4 se presentan y analizan los resultados; y, finalmente, se presentan las conclusiones del estudio.

\section{Definiciones, Clasificaciones y VARIABLES dE anÁLISIS}

Para la Segunda Encuesta de Innovación, las ADT son "todas aquellas acciones llevadas a cabo por la empresa, tendientes a poner en práctica conceptos, ideas y métodos necesarios para la adquisición, asimilación e incorporación de nuevos conocimientos" (Colombia. DANE, DNP \& Colciencias, 2005, p. 23). Según esta misma fuente, estas actividades cubren las siguientes tecnologías:

"Tecnologías incorporadas al capital: Incorporación a la empresa de conceptos, ideas y métodos, a través de la compra de maquinaria y equipo con desempeño tecnológico mejorado (incluso software integrado) vinculado con las innovaciones implementadas por la empresa. Conforma lo que se conoce como cambio técnico "incorporado". Esto constituye nuevos conocimientos adquiridos a través del análisis y uso de nuevos procesos mecánicos, materiales de partes y piezas y en general de nuevos conceptos e ideas incorporadas en la maquinaria (p. 24).

Tecnologías de gestión: La inversión en tecnologías de gestión comprende la adquisición de conocimientos y el procesamiento de información orientados a ordenar, disponer, organizar, graduar o dosificar el uso de los recursos productivos para obtener mayor productividad o competitividad (p. 25).

Tecnologías transversales: Estas inversiones corresponden a la incorporación de conceptos, ideas y métodos como resultado de una actividad de investigación llevada a cabo, ya sea en una forma rutinaria o no, por fuera de la empresa o a pedido de ésta. Estas tecnologías incluyen inversión en actividades de transferencia o adquisición de tecnología, inversión en Tecnologías de la información y la comunicación (TICs), inversión en actividades de Biotecnología y programas de diseño industrial (p. 33).

Proyectos de I+D: Comprenden el trabajo creativo emprendido sistemáticamente para incrementar el acervo de conocimientos, 
y el uso de este conocimiento para concebir nuevas aplicaciones. Pueden incluir el desarrollo de prototipos y plantas piloto. Un proyecto de I+D puede ser de investigación básica, aplicada o de desarrollo experimental (p. 35).

Capacitación Tecnológica: Comprende la capacitación en temas estrechamente relacionados con las tecnologías centrales en el proceso productivo de la empresa. Estas tecnologías pueden ser "blandas" (gestión y administración) o "duras" (tecnología de procesos productivos), que involucran un grado de complejidad significativo (no evidente) que requiere de un personal capacitador altamente especializado (p. 36)”.

En relación con las entidades internacionales de financiación de ADT, las cuales serán objeto de análisis en el apartado 4.1"Reconocimiento de Instituciones Financieras para el financiamiento de ADT", la Segunda Encuesta de Innovación a través del Manuel de Diligenciamiento (Colombia. DANE, DNP \& Colciencias, 2005) las clasifica y define como se muestra a continuación:

"Banca de inversión: Se consideran establecimientos de crédito las instituciones financieras internacionales cuya función principal consista en captar en moneda legal recursos del público en depósitos, a la vista o a término, para colocarlos nuevamente a través de préstamos, descuentos, anticipos u otras operaciones activas de crédito (p. 78).

Banca comercial internacional: Son instituciones financieras con el objeto de realizar operaciones activas de crédito, entre países (p. 78).

Aportes casa matriz: Se refiere a los recursos propios de la empresa provenientes del país de origen de la misma (p. 78).

Organismos internacionales -OEA, ONU, UE-: Se refiere a los recursos provenientes de organismos de cooperación técnica internacional como la Organización de Estados Americanos, Organización de las Naciones Unidas, Unión Europea, etc. (p. 78).

Cooperación internacional: Se refiere a los recursos provenientes de países que tienen programas de cooperación técnica, los cua- 
les se brindan en su mayoría a través de proyectos en diferentes áreas (p. 78).

Programa CARANA: La Agencia Internacional para el Desarrollo del gobierno de los Estados Unidos (USAID) ha asignado a la empresa CARANA la dirección de un proyecto de desarrollo empresarial, que busca incrementar rápidamente los niveles de ventas, empleo y rentabilidad de las pymes colombianas (p. 78)".

Finalmente, las variables tamaño de empresa y tipo de empresa (según el origen extranjero o nacional del capital), que se utilizan para caracteriza los clúster o agrupaciones encontradas en el estudio, se definen como sigue: el tamaño de la empresa depende del número de empleados, siendo microempresas si tienen menos de 10 empleados; pequeñas entre 10 y 50 empleados; medianas entre 51 y 200; y grandes para más de 200 empleados. En cuanto al tipo de empresa, una empresa es considerada extranjera si su capital accionario extranjero es mayor al 50\%; en caso contrario, se considera nacional.

\section{Metodología}

\subsection{0rigen de los datos}

El principal insumo para realizar el trabajo se encuentra conformado por los datos producidos por la Segunda Encuesta de Innovación y Desarrollo Tecnológico (EDIT II) en el Establecimiento Industrial Colombiano, aplicada en 2005 para los años 2003 y 2004. Los datos se obtuvieron por entrevista directa a 6.172 Establecimientos Industriales y tuvo cobertura nacional representativa de agrupaciones industriales según código CIIU. La unidad de selección y observación es el establecimiento industrial definido como la unidad económica que, bajo una forma jurídica única o un solo propietario y en una sola ubicación física, se dedica a la producción del grupo más homogéneo posible de bienes manufacturados. 
La recolección de la información se realizó a través de las Direcciones Territoriales y Subsedes DANE, en un período promedio de cuatro meses (marzo-junio de 2005). Esta encuesta incluyó los establecimientos industriales que ocupan 10 o más personas o que obtengan una producción anual igual o superior a $\$ 65$ millones en el año de referencia.

\subsection{Herramientas para el análisis de datos}

El estudio tiene un primer enfoque descriptivo para analizar las entidades internacionales de financiamiento de ADT, y un enfoque no supervisado de técnicas estadísticas exploratorias de análisis multivariante para la analizar la cooperación internacional en actividades de I+D y Capacitación Tecnológica (CT). Previamente se realizó un preprocesamiento de los datos que consistió en la identificación y tratamiento de outliers o datos atípicos para las variables seleccionadas, así como la construcción de histogramas y BoxPlot.

Las Técnicas de Análisis Multivariante (TAM) se emplearon para la reducción y/o de clasificación de variables (Análisis de Factor y Análisis de Clúster). Estas técnicas fueron útiles en el hallazgo de perfiles y características de grupos de empresas similares.

\section{Resultados}

Los resultados se presentarán a través de dos apartados. El primero es en esencia un estudio descriptivo acerca de las entidades internacionales de financiamiento de ADT. En el segundo apartado se mostrará un estudio de reducción y clasificación de variables mediante TAM.

\subsection{Reconocimiento de Instituciones Financieras para el Financiamiento de Actividades de Desarrollo Tecnológico (ADT)}


Con base en la pregunta de la Encuesta "¿Reconoce o ha escuchado la siguiente entidad internacional de financiamiento?" y la clasificación de las fuentes de financiación presentada en la sección 2 se construye la Tabla 1. Esta Tabla muestra que la visibilidad de las entidades de financiamiento oscila entre el $22.3 \%$ y el $7.2 \%$, valores muy por debajo de lo esperado, lo que revela un alto desconocimiento de las posibilidades de financiamiento internacional de las ADT que ofrecen oportunidades para las empresas. Una posible causa de este desconocimiento podría ser la falta de difusión o publicidad comercial de parte de las entidades financieras para dar a conocer sus productos.

TABLA 1. ReCONOCIMIENTO (VISIBILIDAD) DE LAS ENTIDADES DE FINANCIAMIENTO

\begin{tabular}{|c|c|c|c|c|c|c|}
\hline $\begin{array}{c}\text { ¿Reconoce } \\
\text { la entidad } \\
\text { de finan- } \\
\text { ciamiento? }\end{array}$ & $\begin{array}{c}\text { Banca de } \\
\text { inversión }\end{array}$ & $\begin{array}{c}\text { Banca } \\
\text { internaci }\end{array}$ & $\begin{array}{c}\text { Casa } \\
\text { Matrix }\end{array}$ & $\begin{array}{c}\text { OEA, } \\
\text { NU, } \\
\text { UE }\end{array}$ & $\begin{array}{c}\text { Coopera } \\
\text { internac }\end{array}$ & CARANA \\
\hline Sí & $22.2 \%$ & $22.3 \%$ & $7.5 \%$ & $17.7 \%$ & $8.9 \%$ & $7.2 \%$ \\
\hline No & $77.8 \%$ & $77.7 \%$ & $92.5 \%$ & $82.3 \%$ & $91.1 \%$ & $92.8 \%$ \\
\hline Total & $\mathbf{1 0 0}$ & $\mathbf{1 0 0}$ & $\mathbf{1 0 0}$ & $\mathbf{1 0 0}$ & $\mathbf{1 0 0}$ & $\mathbf{1 0 0}$ \\
\hline
\end{tabular}

FuENTE: A PARTIR DE DATOS DE LA EDIT II

Habiendo señalado lo anterior, de la Tabla 1 cabe también destacar la visibilidad que posee el sistema bancario (Banca de Inversión y Banca Internacional) entre las entidades financiadoras de las ADT, con porcentajes de respuestas positivas cercanas al $22 \%$ cada uno. De hecho, del total de respuestas afirmativas, el sistema bancario posee el $52 \%$ de las respuestas. En contraste, la Cooperación Internacional es prácticamente invisible como fuente de financiación de las ADT, siendo un programa específico, CARANA, casi tan visible como toda la Cooperación Internacional junta.

Más allá de la visibilidad de las entidades de financiamiento, el interés podría recaer en la efectiva utilización de la entidad como 
fuente de recursos para financiar las ADT de la empresa. A este respecto y según los datos de la Encuesta, solo 14 de las 6.212 firmas efectivamente usaron una fuente de financiamiento internacional. En otras palabras, solo el 1\% de las firmas estudiadas utilizaron en algún momento una o varias de las fuentes de financiación mencionadas para las ADT. Como se muestra en la Figura 1, la Banca Internacional se convierte en la vía más utilizada por las empresas para la consecución de recursos $(0,47 \%$ del total de las firmas estudiadas).

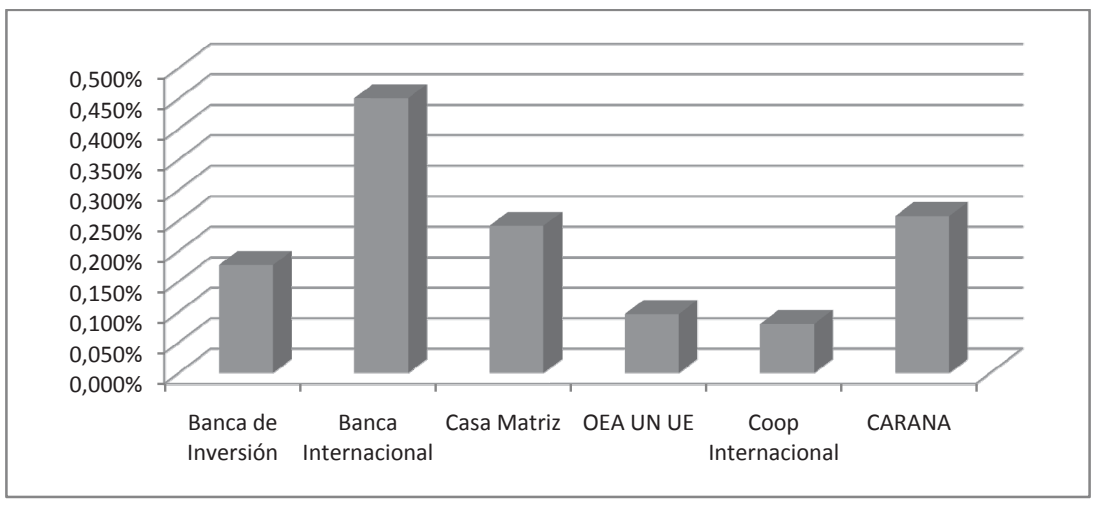

Figura 1. Porcentaje de firmas que utilizaron ReCursos para las ADT PROVEnienTES DE LAS FUENTES FINANCIERAS INTERNACIONALES.

FUENTE: A PARTIR DE DATOS DE LA EDIT II

\subsection{Financiación Internacional de actividades de I+D y de Capacitación Tecnológica (CT).}

Este apartado se enfoca en la financiación de las actividades de I+D y de CT, y al mismo tiempo encuentra el nivel de participación del capital extranjero. La Tabla 2 muestra el porcentaje de dinero que las firmas establecidas en Colombia invierten en actividades de I+D y en actividades de CT. Esta última es, sin lugar a dudas, el enfoque tomado por la gran mayoría de las compañías, puesto que casi la mitad de los recursos reportados se destinan a esta actividad. Como era de esperarse, la investigación básica posee la menor participación con un porcentaje del 3\%. 
TABLA 2. DistribuCIÓn de LAS GAStos En I+D Y CAPACITACIÓN TECNOLÓGICA

\begin{tabular}{|l|c|c|}
\hline \multicolumn{1}{|c|}{ Actividad } & $\begin{array}{c}\text { Total invertido } \\
\text { (US\$)* }\end{array}$ & Porcentaje \\
\hline Investigación básica & 2,948 & $3 \%$ \\
\hline Investigación aplicada & 20,675 & $21 \%$ \\
\hline Desarrollo experimental & 30,195 & $30 \%$ \\
\hline Capacitación tecnológica & 46,692 & $46 \%$ \\
\hline Total & $\mathbf{1 0 0 , 5 1 0}$ & $\mathbf{1 0 0} \%$ \\
\hline
\end{tabular}

* Valores en miles de dólaRes. La CONVERsión SE hizo de ACUeRDo al PROMEdio de LA TASA DE CAMBIO EN LOS AÑOS 2003 Y 2004 SIN AJUSTE INFLACIONARIO.

FUENTE: A PARTIR DE DATOS DE LA EDIT II

Como se presentó en la metodología del trabajo, para el análisis se optó por aplicar un enfoque pasivo o no supervisado, realizando pruebas de reducción de variables y asociación sobre las variables seleccionadas, de modo que se pueda observar qué información y patrones de interés emergen. Se seleccionaron las variables de relacionadas con la cantidad de dinero invertido en actividades de investigación aplicada en el año 2003-2004, cantidad de dinero invertido en desarrollo experimental en el 2003-2004, y la cantidad de dinero invertido en actividades de capacitación tecnológica en el 2003-2004. La variable relacionada con el valor invertido en investigación básica no fue tenida en cuenta por ser un valor nulo en la casi totalidad de establecimientos colombianos (Tabla 3).

Tabla 3. Variables seleccionadas para el estudio

\begin{tabular}{|l|l|}
\hline \multicolumn{1}{|c|}{ Variable } & \multicolumn{1}{c|}{ Código } \\
\hline $\begin{array}{l}\text { Inversión en Actividades de Investigación Aplicada } \\
\text { en el 2003-2004 }\end{array}$ & INVAPLIC \\
\hline $\begin{array}{l}\text { Inversión en Actividades de Desarrollo Experimental } \\
\text { en el 2003-2004 }\end{array}$ & DESEXP \\
\hline $\begin{array}{l}\text { Inversión en Actividades de Capacitación Tecnológica } \\
\text { en el 2003-2004 }\end{array}$ & CAPTECN \\
\hline
\end{tabular}


El propósito es identificar agrupaciones de empresas o clústeres que posean una característica o perfil similar que permita analizarlos y caracterizarlos posteriormente. Primero se realizó un Análisis de Factor para reducir las variables y, posteriormente, un análisis de clúster para identificar las agrupaciones. Para verificar la adecuación entre datos y técnica, se realizó una prueba KMO (medida de adecuación de Kaiser-Meyer-Olkin) y un test de esfericidad de Barlett con su significancia sobre las tres variables presentadas en la Tabla 3 . Los valores de 0,582 y 0,000 se obtuvieron para la prubea KMO y para la significancia del test de esfericidad respectivamente, lo que permite concluir que los datos son adecuados para realizar un Análisis de Factor (KMO > 0,5 y Valor $\mathrm{P}<0,05)$. Al realizar la extracción de los factores por medio del software SPSS, se obtienen dos factores que explican el $76 \%$ de la varianza, por medio de la metodología de los componentes principales. La Tabla 4 presenta la relación encontrada entre los dos factores en relación con las tres variables seleccionadas y relacionadas con las actividades de I+D y de CT.

Tabla 4. MÉtodo de EXTRACción: AnÁlisis de COMPONENTES PRINCIPALES

\begin{tabular}{|l|l|l|}
\hline \multicolumn{1}{|c|}{ Código } & Factor $\mathbf{1}$ & Factor $\mathbf{2}$ \\
\hline INVAPLIC & 0,615 & 0,433 \\
\hline DESEXP & 0,903 & $-0,03$ \\
\hline CAPTECN & 0,05 & 0,946 \\
\hline
\end{tabular}

Método de Rotación: Varimax con Normalización Kaiser (converge en 3 ITERACIONES)

Como lo muestra la Figura 2, el Factor 1 tiene afinidad con las variables relacionadas con los esfuerzos en I+D (Investigación Aplicada y Desarrollo Experimental) a través de los recursos invertidos, mientras que el Factor 2 representa la variable relacionada con la CT. El Factor 1 se etiqueta como esfuerzos en $\mathrm{I}+\mathrm{D}$ el factor 2 como esfuerzos en $\mathrm{CT}$. 


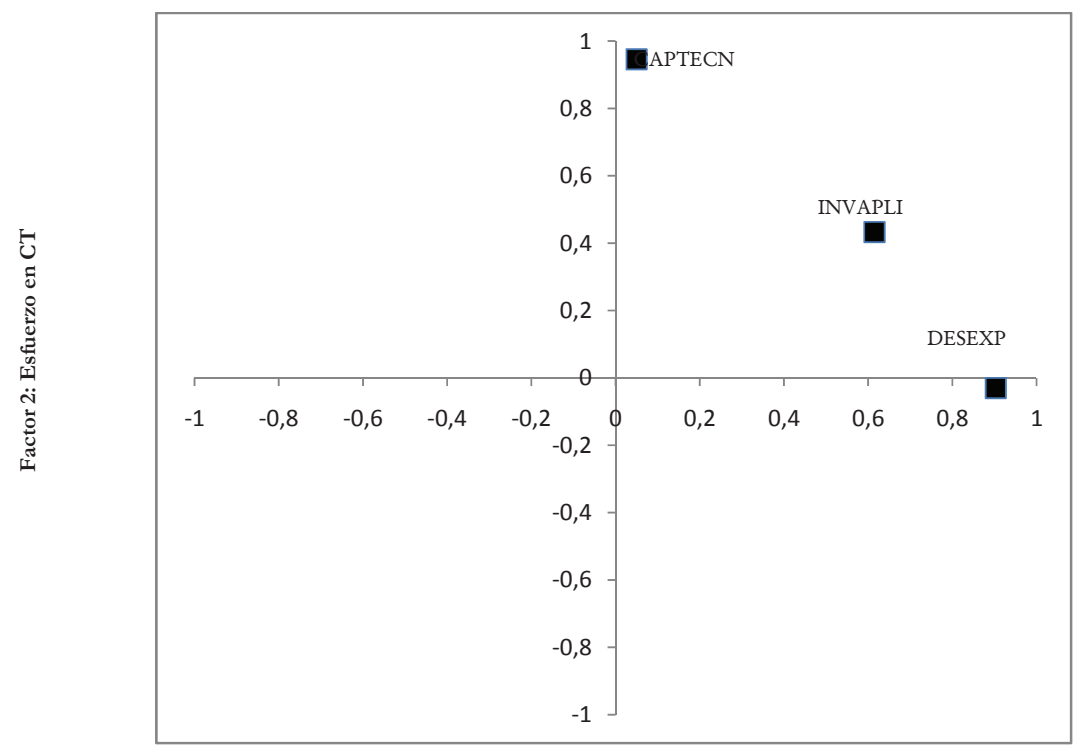

Factor 1: Esfuerzo en I+D

Figura 2: Componentes del AnÁlisis de Factor

Posteriormente se realiza un Análisis de clúster sobre las puntuaciones obtenidas de los dos factores encontrados anteriormente. $\mathrm{Al}$ aplicar el análisis por medio de la metodología de las K-medias, el software SPSS muestra dos clústeres presentados en la Tabla 5 y etiquetados como los Establecimientos Investigadores y los Establecimientos Capacitadores.

TABla 5. Nombre y NúmeRo de EStAblecimientos en CADA ClÚSter

\begin{tabular}{|l|c|}
\hline $\begin{array}{c}\text { Nombre del } \\
\text { Clúster }\end{array}$ & $\begin{array}{c}\text { Numero de } \\
\text { Establecimientos }\end{array}$ \\
\hline Investigadores & 20 \\
\hline Capacitadores & 6196 \\
\hline Total & 6216 \\
\hline
\end{tabular}


Clúster 1: Los Establecimientos Investigadores

Estas firmas se caracterizan porque realizan las mayores inversiones en actividades de I+D y de CT respecto al total de la muestra. Solo corresponden al 0,32 \% del total, lo que evidencia la poca iniciativa que tiene la empresa promedio para realizar actividades investigativas. Estas empresas poseen en promedio 1.275 empleados, por lo que es un clúster conformado principalmente por grandes empresas. El 40\% son empresas cuyo capital extranjero es mayor o igual al 50\% de la composición accionaria, y el $60 \%$ son empresas de capital mayoritariamente nacional, lo que muestra un amplio predominio relativo de las empresas con capital extranjero frente a las de capital nacional, teniendo en cuenta que el número absoluto de éstas es muy superior.

Los recursos obtenidos para financiar actividades I+D provienen principalmente de fuentes nacionales, a excepción de dos empresas de las 20, de las cuales una utilizó recursos provenientes de Alemania para realizar Investigación Aplicada y la otra recursos provenientes de los Estados Unidos para Investigación Aplicada y Desarrollo Experimental. En otras palabras, los Establecimientos Investigadores se caracterizan por no utilizar recursos provenientes del exterior, sino de fuentes nacionales, para financiar sus actividades investigativas.

Clúster 2. Los Establecimientos Capacitadores

Casi todas las empresas de la muestra pertenecen a este clúster (99.7\%), por lo que sus características son representativas del establecimiento industrial colombiano. Este clúster, a diferencia de los Establecimientos Investigadores, no gasta cantidades importantes de dinero en actividades de I+D ni en CT. Sin embargo, la mayoría de las firmas que pertenecen a este clúster tienen alguna inversión en actividades de CT, obviamente sin llegar alcanzar los niveles encontradas en los Establecimientos Investigadores. Es decir, este clúster muestra que la firma promedio en Colombia no invierte significativamente en $\mathrm{I}+\mathrm{D}$ y solamente de forma tímida en actividades de CT, de donde proviene el nombre de Establecimientos Capacitadores. 
En promedio, las empresas de este clúster están conformadas por 81 empleados, evidenciando que es un clúster representativo de la PYME colombiana. Solo el 5\% de las firmas poseen un capital accionario extranjero mayor o igual al 50\%, mientras que el $95 \%$ son consideradas empresas nacionales.

A semejanza de los Establecimientos Investigadores, los cuales mostraron una participación muy pobre en el financiamiento extranjero de sus actividades, el clúster de los Establecimientos Capacitadores financia sus actividades con recursos de fuentes nacionales (alrededor del 93\% de las empresas). Cerca del 97\% de los recursos invertidos provienen de tales fuentes y solamente el $7 \%$ de las empresas del clúster utilizaron recursos de fuentes extranjeras para financiar sus actividades.

La Figura 3 presenta un desglose del 3\% de los recursos financieros provenientes de fuentes extranjeras, según su país de procedencia y el número de empresas financiadas. Se observa el predominio estadounidense (24 empresas financiadas), seguido de Cabo Verde y, más atrás, de Alemania y Brasil. Las fuentes financieras para las demás empresas están dispersas en un número alto de países, cada uno financiando menos de cinco empresas.

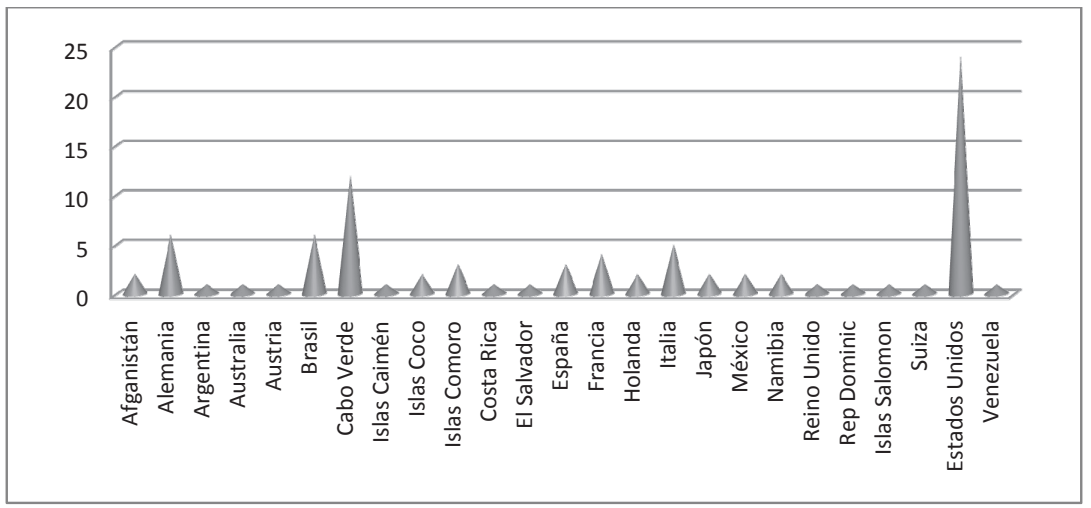

Figura 3. NúmeRo de ESTABleCiMientos finANCIAdos POR PAís

Estados Unidos no solo es el país que más número empresas financia del clúster de Establecimientos Capacitadores, sino que 
también es el que más recursos aporta respecto a los demás países. La Figura 4 ilustra esta situación, en la que se aprecia que los Estados unidos aportan alrededor del 30\% del total de los recursos del clúster provenientes del exterior.

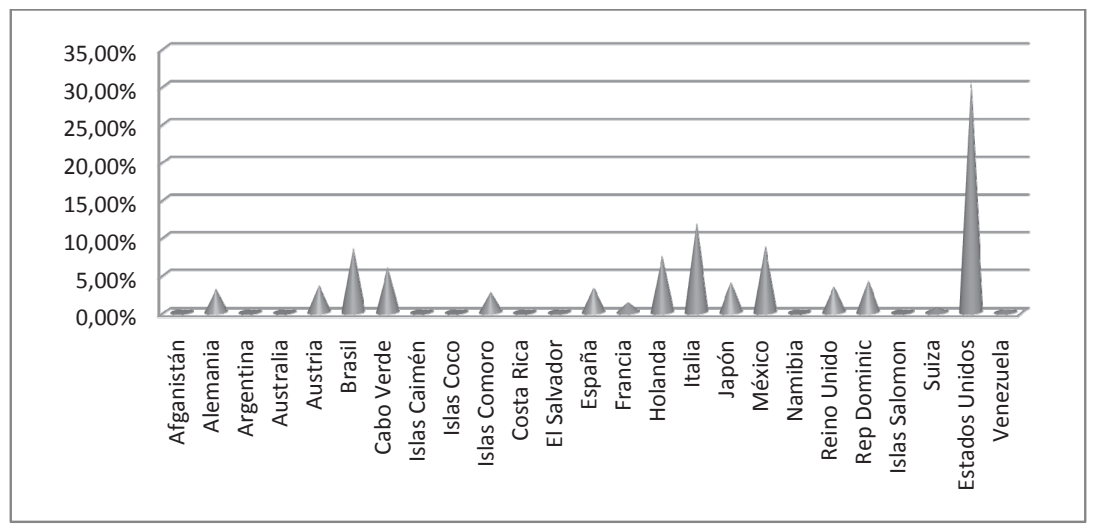

Figura 4. PorcentaJe de La FINANCIACIÓN TOtAL EXTRANJeRA POR PAís

Aquí, de nuevo, se observa la amplia dispersión de los recursos según se fuente, descontando el aporte estadounidense. Sin embargo, si se consideran los países europeos en conjunto (Alemania, Austria, España, Francia, Holanda, Italia, Suiza e Inglaterra), la participación sería muy relevante y sobrepasaría incluso a los Estados Unidos en el aporte extranjero para la financiación de actividades de I+D y Capacitación Tecnológica.

\section{Conclusiones}

Respecto a la financiación internacional de las ADT de las empresas estudiadas y considerando las fuentes previamente identificadas, el trabajo evidencia un acceso muy limitado a los recursos provenientes de tales fuentes, con sólo el 1\% del total de las empresas reportando uso de por lo menos una de las fuentes. Este resultado descriptivo muestra un escaso flujo de recursos financieros del exterior hacia Colombia para este tipo de actividades. 
Esta situación se corresponde con la baja visibilidad de las entidades financieras internacionales como fuentes de recursos para ADT. De esta visibilidad, la mayor parte se dirige al sistema bancario (Banca de Inversión y Banca Internacional), siendo también notable la Cooperación Internacional, pero por el hecho de que es prácticamente invisible como fuente de recursos empresariales para ADT.

En resumen, las ADT de las empresas colombianas están escasamente internacionalizadas en lo que respecta al flujo de recursos financieros externos. Este hecho, junto con la pobre visibilidad de las fuentes internacionales de recursos y la baja intensidad de las actividades empresariales de I+D y $\mathrm{CT}$, configuran un escenario que debe ser motivo de examen y tratamiento en la doble perspectiva de la política pública y la gestión empresarial.

Por otra parte, el estudio muestra que los recursos de financiación de actividades de I+D y de CT provienen principalmente de fuentes nacionales, tanto para el clúster de los Establecimientos Investigadores como para el de los Capacitadores. Sin embargo, las actividades de CT en este último clúster generan una financiación internacional mucho más fuerte y diversa en sus fuentes que la encontrada para el clúster de los Investigadores.

Las fuentes de financiamiento internacional de actividades de CT para el clúster de los Establecimientos Capacitadores se sitúan principalmente en los Estados Unidos y Europa (como suma total de los países que la componen). Sin embargo, como país individual, los Estados Unidos poseen un liderazgo en número de firmas financiadas y en porcentaje sobre el total financiado frente a los demás países, los cuales muestran una amplia dispersión en número y una escasa participación en empresas financiadas y recursos aportados.

De los dos clústers encontrados, los Establecimientos Investigadores se caracterizan por su esfuerzo en I+D, el tamaño grande de sus empresas y la alta proporción de capital accionario extranjero. Estas, sin embargo, no son características representativas de la muestra de empresas del estudio. Esta representatividad recae en 
el clúster de Establecimientos Capacitadores, que invierten muy poco en I+D y tímidamente en CT. El tamaño de sus empresas es predominantemente pequeño y mediano, y su composición accionaria está dominada por capital nacional.

\section{Reconocimientos}

El presente trabajo fue financiado por el Instituto Colombiano para el Desarrollo de la Ciencia y la Tecnología (COLCIENCIAS) a través del Proyecto DESCUBRIMIENTO.

\section{ReFerencias}

Beers, Cees van, Berghäll, Elina \& Poot, Tom (2008) R\&D internationalization, R\&D collaboration and public knowledge institutions in small economies: Evidence from Finland and the Netherlands. Research Policy, 37, 294-308

Behrman, .I. N. and Fischer, W. A. (1980) Overseas R\&D R. Nelson, G. Silverherg and L. Soete, pp. 496527. Activities of Transnational Companies. Oelgeschlager, Pinter Publishers, London and New York. Gunn \& Hain, Cambridge, MA.

Colombia. DANE, DNP and Colciencias (2005). Manual de diligenciamiento de la segunda encuesta.

Colombia. COLCIENCIAS (2008). Colombia construye y siembra futuro. Política nacional de fomento a la investigación y la innovación.

Dunning, J. H. (1992) Multinational enterprises and the globalization of innovatory capacity. In Technology Management and International Business: Internationalization of R\&D and Technology, ed. 0. Granstrand, L. Hakanson and S. Sjölander, pp. 19-51. John Wiley \& Sons Ltd., Sussex, England.

Europe Commission (2007). Europe in the global research landscape. P. 113. Website http://publications.europa.eu

Freeman, C (1993). The Economics of Industrial Innovation. 3rd edition. Londres.

Grosse, Robert. R\&D by TNCs in Latin America. Article in press 
Hidalgo, Antonio; Fernández, Celia; López, Juan (2002). Evaluación de los 10 de años de IBEROEKA. Fundación General de la Universidad Politécnica de Madrid

Katz, R., Rebentisch, E.S., Allen, T.J., (1996). A study of technology transfer in multinational cooperative joint venture. IEEE Transactions on Engineering Management 43 (1), 97-105.

OCDE (2005) Oslo Manual - Guidelines for collecting and interpreting innovation data. 3rd Edition. European Commission, Organization for Economic Co-operation and Development, Statistical Office of the European Communities, Paris.

OCDE (2008). Recent trends in the internationalization of R\&D in the enterprise sector. Special session on globalization. Organization for Economic Cooperation and Development, Paris.

Reddy, Prasada (1997). New Trends in Globalization of Corporate R\&D and Implications for Innovation Capability in Host Countries: A Survey from India. World Development, Vol. 25, No. 11, pp. 1821-I 837.

Richards, Malika and Yang, Yi (2007). Determinants of foreign ownership in international R\&D joint ventures: Transaction costs and national culture. Journal of International Management, 13, 110-130.

Schumpeter, J (1939). Business cycle: a theoretical and statistical analysis of the capitalist process. Mac Graw Hill.

Vonortas, N.S., 1997. Research joint ventures in the US. Research Policy 26, $577-595$. 